lial web or of the fungous mass as it comes from the ear,
will, if placed in a glass box, continue to grow and to rewill, if placed in a glass box, continue to grow and to re-
tain the microscopic appearance of Aspergillus nigric ins.
From the fact of this retention of form, it would seem From the fact of this retention of form, it would seem
that this is a specific fungus, and not ordinary mould modified by growing in the ear.

It may be said, then, that in general there are found with the aid of the microscope, four distinct elements in
a fungous mass of aspergillus taken from the human ear,

viz. : The mycelial web, composed of the so-oalled tubules, rootlets, or filaments.
2 . The fruit stalk or hyphen, and

3. Its head or the sporangium.
3.

4. The free spores.

The first, the filaments or tubular rootlets, are composed component cellules are from $0.020 \mathrm{~mm}$. to $0.090 \mathrm{~mm}$. in 'The fertile hyphens or fruit stalks vary in diameter from
$0.009 \mathrm{~mm}$. to $0.013 \mathrm{~mm}$., and their length is $0.770 \mathrm{~mm}$. These terminate in the so-called receptaculum, the placenta
of Micheli, which has a diameter of $0.028 \mathrm{~mm}$. (Robin.) On this grow the sterigmata and spores, the three together forming the so-called capital or head, $0000 \mathrm{~mm}$. in diamThe spores are spherical and measure $0.003 \mathrm{~mm}$. in diameter. By careful fine adjustment of the lens, their surface is seen to be echinate. Some idea of the immense number
of these spores in any case may be gained by Paccini's estimate, quoted by Robin, that there are nineteen thousand spores on each fruit head.

Macroscopic features. - The macroscopic appearances of a
mass of this fungus, as found in or washed from the ear are worthy of attention. For, although the microscope must in every case decide the presence of aspergillus in a
suspicious object coming from the external auditory canal, suspicious object coming from the external auditory canal,
neverthelessethe microscopic features of a plug or membrane composed of this aural fungus are of a nature to lead the composed of this aural fungus are of a nature to lead the
surgeon to suspect that he has to deal with a case of aspergillus in the ear. If an ear containing a mass of aspergillus be present most usually an appearance which leads to the sup-
position that the ear is occluded not by wax, but by a foreign matter of an organic nature.

If the fungus has not been growing long in the ear, mere ly a patch of pale yellow, pollen like inatter, of varying dianeters, will be detected at the fundus of the auditory
canal. This small colony of spores just developing into filaments, for such it is, is usually situate on the membrana tympani or very near it. In any case, whether the first
deposition of spores occurs there or not, the tendency of the aspergillus is to grow over the drum head first, and from
that point it spreads outward, covering the wall of the
meatus, until a hollow cast of the canal is formed by the vegetable parasite. The pollen-like appearance is seen only in the very earliest stages of a growth of that which is

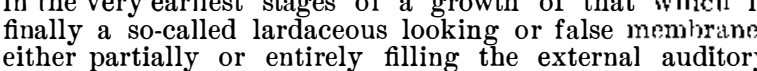
canal.

In some cases the fungous mass looks like a ball or plug of wet newspaper, and in others the ear may seem to be
plugged with a substance looking like wool. An inexperiplugged with a substance looking like wool. An inexperi-
enced eye might conclude that the occluding plug this
formed is of ear wax; but ear wax looks more solid, slin. formed and drier, and it never excites pain and intlarnmatio in the ear like the fungus aspergillus.

An that mass of aspergillus does not lose its coherence when sub
jected to immersion in water or glycerine; but a ling of throughout the water or any other fluid in which it is allowed to lie. such a mass washed from the ear were aspergillus, or the peculiar laminated, epithelial plug, the so-called Keratosis

[We omit the rectal of the cases, a large number of which GENERAL REMARKS.

Symptoms. - The symptoms of the growth of this para
sitic funcus in sitic fungus in the external ear may be briefly given as sting
ing, itching, with dullness of hearing, some pain, and a watery but scanty discharge. The patient may finally com should become inflamed

An ear thus affected will show on examination, the pres An ear thus affected will show on examination, the pres-
ence in it of a grayish, sometimes mottled, flaky mass, or if ence in it a a grayish, sometimes mottled, flaky mass, or if
examed in the earliest stages of disease, it reveals the
presence of a false membrane neatly adapted to the membrana tympani and the inner part of the auditory canal
From this point the false membrane may- sometimes doesFrom this point the false membrane may-sometimes does-
extend along the canal until ii. projects from the orifice at the concha of the auricle. When seen in its early stages of growth, the false membrane over the drum head looks dr and resembles a piece of wet newspaper. Any free edge of of epithelium. Once seen, this parasitic membrane is easily
recognized again. The diagnostic features of such a false recognized again. The diagnostic features of such a false
membrane or fungous mass may be learned from what has

ances
If any doubt should exist about the presence of aspergillu
in the ear it is usually dissipated in a day or two, on the re turn of the patient, for unless the treatment has been the
proper one and very successful, the false membrane will proper one and very successful, the false membrane will
have formed again. This, of course, removes all doubts, and should prompt us to great activity and watchfulness, or The microscope will remove all doubts as to the nature of any suspicious looking flake or lump removed from the

Etiology.-It must be evident to the reader of the details of the twenty cases here presented, that this disease is not confined to those living in poverty and squalor. In fact my
experience is the reverse of this. Only three cases could be said to have occurred in the poor and unclean. As long as
the secretion of ear wax is unimpeded and, when secreted, the secretion of ear wax is unimpeded and, when secreted,
if the cerumen is not scraped away, no one is likely to be if the cerumen is not scraped away, no one is likely to be
affected by aspergillus in the ear. If the ceruminous secrc-
tion is disturbed in any way, especially if now the skin of the auditory canal be abraded, anybrowy may be, probably will be, attacked by this parasite. The chief causes of the
growtll of this parasitic fungus in the ear, I find to be ungrowtli of this parasitic fungus in the ear, I find to be un-
due picking and scratching of the ear, and dropping in and leaving there oils and fats of various kinds and pieces of
vegetable matter. These becoming rancid or putrescent, a
fitting soil for the aspergillus is given. Next to these causes is the neglect to wash the ear after it
as been the seat of boils or any inflammation, which may eave behind it small particles of pus, serum, blood, etc. It If the patient should be living in damp apartments, of
ecome the nidus of a colony of aspergillus. course this must be ended, if possible, either by cleansing
and drying his dwelling, or by removal from this probable source of disease. If any other excitant can be shown to be I

I must repeat here what I have written elsewhere concerning the protective function of cerumen. There is no evi dence that the aspergillus grows on the natural ceruminous
secretion of the auditory canal. It appears, indeed, that but for the presence of the cerumen in the canal, the ear me latter seeks a secluded spot for growing. The protection
then of the cerumen in this particular is shown in the fact that
in an ear, the canal of which is sheathed with ear wax, asin an ear, the canal of which is sheathed with ear wax, as-
pergillus is rarely found at all, and never in a flourishing ondition, while in an ear invaded by aspergillus, cerume
sarely, if ever, found in a normal quantity or condition. On this point of contagion I am very careful, for it would
On e very easy to convey aspergillus from one person to
nother, not only by the syringe, but by specula, cotton

Treatment.- The treatment of aspergillus in the ear con-
ists in killing and removing all parts of the plant, and especially all its germs. The syringe is the best means of re-
moving the parasite, after it forms into false membrane, but oving the parasite, after it forms into false membrane, but
the latter be adherent, other mechanical means may be necessary. Hence I find it useful, if the fungous membrane
as reached any size, or if it be visible as whitish flakes or pots, to wipe these traces of it away from or off the walls ndts, to wipe these trangani, by means of the cotton dossil on
the cotton holder. This is easily done and causes no pain
to the patient. If these patches slowld be very adherent, the patient. If these patehes sllould be very adherent,
they must not be forcibly' removed. They are to be loosenhey must not be forcibly removed. They are to be loosen-
ed then either by time or by the use of a parasiticide. Experience shows me that these false membranes are not
sually adherent but easily detached. I have never em. ployed but two parasiticides, viz., alcohol, usually pure, but sometimes in various proportions with water, rarely veaker than one of
posulplite of soda.

My solitary experience with hyposulphite of soda, three rains to the fluid ounce of water, leads me to place it next
to alcohol, as a destrover of the aspergillus. In the future propose to use it more frequently than in the past. I have
prove re to destroy the parasite, without subsequent syringing on his part. Of course this omission of syringing on the an aurist daily. The greatest gentleness is requisite in all in all these cases the ear seerns to possess a readiness to slip Of the eczematous state.

Of course, should this complication arise in the parasitic
disease. it must be combated on general principles. If pos-
sble; all fatty matter should be excluded from the treatment sible; all fatty matter should be excluded from the treatment
of eczema in these cases, since oleaginous substances feed the fungus. *
In conclusion, it may be said the ear thus diseased should be caref ully examined by means of the ear mirror and
speculum every day, and the treatment modified according speculum every day, and the treatment modified according
to the stages of the disease. The least irritation of the ear,
combined with the most efficient destruction and mechanical combined with the most efficient destruction and mechanical
removal of the parasite, will give the most satisfaction t
both surgeon and patient.

\section{DETERMINATION OF POTASSA AND SODA}

By W. KNop and J. HAZARD.

THE authors dissolve in hydrofluoric acid, evaporate,
drench the residue with concentrated sulphuric acid, tho removing the bulk of the silica as silicon fluoride. The sul
phuric acid is then evaporated off the dry rosidue with five or six drops of concentrated sulphuric acid, heated drenched with 150 c.c. water, and barium hydrate added till
red litmus paper is turned distinctly blue. The mixture of barium sulphate, silica, alumina, magnesia. and ferric oxid rated to dryness, adding, when it is concentrated down about 209 c.c., a few grammes of dry ammonium sesqui-
carbonate. When perfectly dry the residue of barium ind calcium carbonate is extracted successively fifteen times, filtered through a smaal filter of 3 to 4 c.m. diameter into a platinum capsule, and evaporated to dryness. The residue is drenched again with 20 c.c. water, the water is decanted
through a similar fresh filter, and the solution-after it has

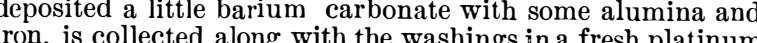
capsule. The alkaline carbonate, to which a few more grasules of ammonium carbonate have been added, is again
'The liquid is then water, observing that no residue remains. The liquid is then neutralized with hyltrorhloric acid, evapo-
rated, the chlorides dried strongly, and the potassium and sodium separated by means of platinum chloride.-Chem

\section{FERRIC HYDRATES}

By Dr. D. Tommasr.

By DR. D. TomMAsI.
IT appears that all these hydrates may be arranged in two
Rural Home a statement of his excellent management. He series, respectively isomeric, the red and the yellow. The former are obtained by precipitating a ferric salt by potassa,
soda, or ammonia. When calcined they present the phe-
nomenon of incandescence they dissolve readily even in the nomenon of incandescence; they dissolve readily, even in the
weakest acids. Ferric chloride dissolves them in quantity, and the solution gives a precipitate of ferric hydrate on the
addition of potassium sulphate or sulphuric acid. Theyare addition of potassium sulphate or sulphuric acid. Theyare
dehydrated on boiling with water. The yellow hydrates are obtained by oxidizing ferrous hydrate or carbonate, or
magnetic hydrate. If these hydrates are calcined they do not display incandescence; they are sparingly soluble in acids,
whether dilute or concentrated; they are not attacked by whether dilute or concentrated; they are not attacked by
ferric chloride; if boiled in water they only lose two mole* The of water, retaining the third, even if boiled in a con* The investigations of Bezold, of Mumich, have fully confirmed th
act that the use of oleaginous matters for the cure of ear diseases is ver entrated solution of calcium chloride. It is possible that and aluminum, may exist in these two modifications. If the two ferric chlorides are treated with silver nitrate, the toms at first and the two others afterwards. In a similar manner the blue chromium chloride gives up all its chlorine
at once, whilst the green chloride gives up two thirds at at once, whilst the green chloride gives up two thirds at
first and the remainder afterwards. There ought to exist phates. The two latter are distinguished not merely by their color, but by the fact that the blue sulphate gives up all it ACTIVE MATTER OF MALT, OR MALTIN AND

By M. Dubrunfaut.

THE author ascribes the following properties to the diastase of Payen. It exists in malt to the extent of one to two
thousandths; it liquefies 2,000 times its weight of starch; it is free from nitrogen, and saccharifies 2,000 times its weight of starch (according to Guerin only 15 times). It has no anhydrous alcohol, and not affected by this liquid. It is not altered on solution in water at $75^{\circ}$, but is modified at $100^{\circ}$. Its maximum of activity is at $75^{\circ}$. Maltin, on the other hand, tains from 0.07 to 0.08 of nitrogen, saccharifies 100 times weight of starch, and possesses a strong lævo-rotatory noluble in, though modified by, strong alcohol. Its maximum activity is below $50^{\circ}$, and it
als, but in many potable waters.

\section{TEST FOR TRACES OF MERCURY} By Ed. Teuber.

THE material, dry and finely powdered,is mixed with well laced in a crucible upon a lisyer of red The mixture is with a layer of iron filings. Y'he lid of the crucible is so must impinge upon the bottom of a small gold capsule

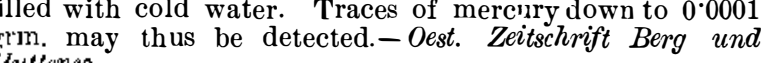

\author{
[Country Gentleman.] \\ HORTICULTURAL NOTES. \\ CURL IN THE PEACH.
}

Prof. BURRILL, of Illinois, has recently made a thorough examination of the minute fungus which had long since Ascomyces deformans. He has traced this fungus to the un-
opened buds. As the leaf expands it is carried along, and bark of a diseased twig is filled with the threads of the fungus, and is sometimes distorted with ridges and swellings mends the pruning away in winter of such twigs as may be Tound to be thus diseased, and committing them to the fire.
To this we would add the importance of keeping the trees in a vigorous state of growth, as feeble ones recover less spicuous after a cold snap just after the leaves are expanded this condition of the weather favoring the development of viously within the stalks.
LATE PEACHES.

The importance of a continuous supply of peaches through the entire season of the year, while they may be had, is obvi-
ous to every one; and now that several very early varieties he been raised, we may have, with a little care, plenty of in autumn, in lititucie as far north as Roclester. Much in quiry bas been made for yood late sorts. M. B. Bateham ciety, gives, as the result of much observation, the follow ing names: The Smock, quite reliable in Northern Olio,
has been widely planted there, and, we may add, ripens woll has gone, and before the ripening of the Smock, he recommerids Hall's Chili, which is a few days later than Craw-
mater ford's Late. This variety originated in Western New York,
but apperars to succeed better in Obio and Michligan. Next after H[ill's Chili he names the Brandywine of New Jersey,
which slightly precedes Smock, while it exceeds it in size which slightly precedes Smock, while it exceeds it in size
and fine arpmearance. The Salway follows the Smock, and does well a little farther south.
EARLY FREESTONE PEACH.

Nearly all the very early varieties which have been strong adhesion of the melting flesh to the stonc; $a$ and one in which the flesh separates freely from the stone has been sought. The Canadian Horticulturist describes a new sort,
known as the Early Canada, in which this desirable characteristic is quite distinct. The fruit ripens about the first of August, possibly a little after the Amsden, and it is described
as of fair size (rather indefinite), bright in color, of excellent as of fair size (rather indefinite), bright in color, of excellent
quality, and nearly a perfect freestone. It is certainly

\section{GOOD CULTUPE OF AN ORCEARD.} a thorough cultivation with a two-horse cultivator the following spring and early in the summer. The fruit the past
season was larger and finer in appearance, and, what is still season was larger and finer in appearance, and, what is still
more important, was less injured by the codling worm. No attempt was made to plow close to the trees. as not being necessary, and avoiding injury to the bark. The quick
grass and thistles in the orchard were nearly extirpated by the treatment thus given. Mr. G. remarks: "Old apple trees exhibit marvelous vitality under proper treatment, and may be made attractive in appearance by pruning." To ing neglected in grass, restored to great productiveness by and copious top-dressing of manure. states that the name appropriately given to this fungus is the fungus, just as certain kinds of weather in summer de-
velop the rust in wheat, the young rust plants existing preIn the autumn of 1878 he plowed it quite shallow, and gave nearly half their branches or shoots were dead when stand- 